\title{
Control of Storage Elements in an Islanded Microgrid with Voltage-Based Control of DG Units and Loads
}

\author{
Tine L. Vandoorn, Jeroen D. M. De Kooning, Bart Meersman, Brecht \\ Zwaenepoel \\ Dept. of Electrical Energy, Systems \& Automation, Ghent University, \\ St-Pietersnieuwstraat 41, 9000 Gent, Belgium, +32 926434 22, \\ Tine.Vandoorn@UGent.be (corresponding author)
}

\begin{abstract}
Because of their small scale and large share of intermittent power sources, islanded microgrids require additional means of flexibility, such as dedicated storage. For controlling the loads and distributed generation (DG) units in an islanded microgrid, the voltage-based droop (VBD) control has been developed. This controller determines the switching actions of these units' power electronic interfaces in order to ensure a stable islanded microgrid operation. In this paper, the VBD control is extended for storage applications, taking into account the state of charge. As the VBD control automatically fixes the priority of power changes of the microgrid elements, the storage elements are included in this priority list without inter-unit communication, benefiting the coordinated integration of DG units in the system. Simulation examples are included illustrating the transient and dynamic response of the VBD control of all grid assets. The paper also provides an experimental validation of the VBD control for both the DG units and the storage elements in an islanded microgrid.
\end{abstract}

Keywords: distributed generation, droop control, microgrid, storage control

\section{Introduction}

There is an urgent need for more flexibility in the electric power system because the electric grid lacks a substantial storage capacity. Nowadays, the balancing of the power system faces some problems. Firstly, it is hard to impossible to accurately predict the demand, the occurrence of incidents on the grid and the renewable energy output. Secondly, the load and renewable generating units are to a large extend inflexible, i.e., they do not react on changes in the power system. Instead of taking the conventional approach of 
investing in grid assets, which requires long lead times and massive investments, flexibility is increasingly being sought elsewhere, e.g. by dynamically controlling the DG units and loads.

Another means for providing more flexibility can be given by storage equipment. For instance, in [1], the operational benefit of a microgrid with wind farm and pumped hydro storage facility is maximized through optimal scheduling in the electricity market. Storage is regarded as not yet economically viable, however, in the future, this may not be the case any more. Even more, not only the economic impact, but also the reliability and environmental assessment should be taken into account for evaluating a microgrid with storage facilities [2]. The potential of energy storage to provide voltage regulation and peak shaving is increasing, e.g., using batteries for supporting the operation of the distribution grids gains wide interest. Even more so than in large interconnected systems, in small islanded systems, energy storage is an important asset. The electrification of autonomous systems often deals with high production costs combined with low quality of supply. The paper [3] estimates the mean annual cost reductions by using energy storage for primary reserve and peak shaving in two isolated Spanish power systems. Also, the optimum sizing of energy storage systems for islanded systems has been widely discussed $[4,5]$.

On one hand, dedicated storage equipment can be installed in the networks. A first, and often-used example are pumped storage hydro facilities, but the available locations for these units are restricted due to geographic issues. Flywheels and battery banks also provide opportunities for dedicated storage. On the other hand, some already installed grid assets can be used as storage equipment, where the storage property becomes an ancillary service. In this sense, electrical vehicles provide huge opportunity. They can either be used as buffer elements, altering their charging times based on the state of the network, which is analogous to demand response. Or, they can be used as storage element with bi-directional power flow.

Microgrids are widely regarded as pilot versions of the grid of the future and fundamental building blocks of the smart grid [6]. Microgrids consist of an aggregation of distributed generation (DG) units, (controllable) loads and often also storage elements [7]. Many microgrid elements are powerelectronically interfaced to the network. Hence, the microgrid control entails the converter control, an overview of which is given in [8]. Microgrids can operate both in grid-connected mode and islanded mode [9]. In the gridconnected mode, the microgrid offers economic benefits for its participants by allowing them to participate in the electricity markets more easily and beneficially. For the network operators, grid-connected microgrids can be seen as single entities, providing them scaling benefits in their system management. Although the normal operation of the microgrid is generally the grid-connected mode, microgrids can island during special circumstances. In 
case of problems in the utility network, such as grid outages or low power quality, microgrids can offer a significant reliability improvement. By operating in the islanded mode, microgrids can also enable maintenance of the grid assets without de-electrifying the network downstream, which is demonstrated in Canada [10]. Also, remote microgrids can help electrifying areas that otherwise have no grid access. This is a significant contribution of microgrids as it is often emphasized that electrification is an important condition for achieving a sustainable economic development.

Even more so than in the conventional electric power system, storage elements are fundamental components of a microgrid, in order to maintain stability, facilitate integration of renewables and improve power quality [11]. Energy storage application in microgrids also provides a test environment for storage extended to the utility grids.

The authors have analyzed the microgrid islanded operation and proposed a control strategy for the DG units as well as the loads. This strategy, called the voltage-based droop (VBD) control, is a primary controller, hence, it is responsible for the reliability of the microgrid [12]. Therefore, it operates fast and automatically. Further issues, such as economic optimization or taking into account the long-term constraints of the grid elements are handled in an overlaying secondary controller [13]. The VBD controller uses the grid voltage as the non-conventional parameter for the active power sharing between the different DG units. The reason for using the voltage amplitude as trigger is the low inertia available in the considered low-voltage islanded microgrids and the high $R / X$ values of the distribution lines.

In this paper, storage equipment will be utilized in addition to the DG and load control. An analogous control strategy for the storage equipment is presented, based on voltage droops and constant-power bands like the VBD control. This enables an optimal coordination of the responses of the different grid assets. After a load change, an automatic priority is set, such that for instance first the controllable DG units, later the storage elements, then the controllable loads and last the other grid elements alter their output/input power. A main advantage is that this priority is followed without inter-unit communication between the units, benefiting the reliability of the microgrid. As the paper presents a generic control strategy, it does not consider the specific energy storage technology, such as batteries and flywheel energy storage, and modeling. An overview of this is given in [11]. In $\S$ II, the VBD control for the DG units and loads is summarized. In $\S$ III, the VBD control for storage equipment is presented. Some simulation examples, including a transient study $(\S \mathrm{IV})$ and some dynamical studies $(\S \mathrm{V})$ are discussed. Finally, $\S$ V presents some experimental results of firstly, the VBD control of the DG units in an islanded microgrid, and secondly, that of the storage equipment. 


\section{Voltage-based droop control: overview}

\subsection{VBD control of $D G$ units}

A survey of various primary control strategies for islanded microgrids is given in [14]. For (extended) islanded microgrids, the droop control concept is most promising to ensure a reliable system operation. Droop controllers in microgrids are derived from the well-known active power/grid frequency $(P / f)$ droop control principle incorporated in the large central synchronous generators in the conventional electric power system [15, 16]. Different variants on $P / f$ droops have been developed, such as adaptive droops [17] and virtual impedance loops $[18,19]$. While the $P / f$ droop control-based methods work well in a microgrid with mainly inductive line impedances, it leads to a concern when implemented in a low-voltage microgrid, without significant inertia and with predominantly resistive lines [20]. In case of mainly resistive lines, the active power $(P)$ is linked with the voltage difference $(V)$, while reactive power $(Q)$ is linked with the phase angle, hence frequency $(f)$. This leads to $P / V$ and $Q / f$ droops as opposed to the conventional $P / f$ and $Q / V$ droops [21, 22].

The voltage-based droop (VBD) microgrid control (Fig. 1) of [12] for islanded microgrids is a variant of the $P / V$ droop control that focuses on an optimal integration of renewables in the network and also incorporates a dc-bus controller. As it is a local primary controller operating without communication, the VBD controller is responsible for the reliability of the system. This VBD controller takes into account the lack of inertia, resistive lines and high share of renewables which are specific characteristics of the considered low-voltage islanded microgrids. It offers high flexibility to address different kinds of distributed energy resources to ensure a stable microgrid operation. As discussed further in the paper, these units are addressed in a predefined priority order, without need for communication. This control goes beyond the state of the art that uses the fit-and-forget approach for integrating DG, which poses limits on the number of renewable sources in the network. In the VBD control, the $P / V$ droop controller is divided into two droop controllers, the $V_{\mathrm{g}} / V_{\mathrm{dc}}$ and $P_{\mathrm{dc}} / V_{\mathrm{g}}$ droop controllers, with $V_{\mathrm{g}}$ the rms value of the terminal voltage, $V_{\mathrm{dc}}$ the dc-link voltage and $P_{\mathrm{dc}}$ the dc-side power as depicted in Fig. 2. The $V_{\mathrm{g}} / V_{\mathrm{dc}}$ droop controller is responsible for balancing the ac and dc-side of the inverter. A difference in dc-side and ac-side power is reflected in a change of the dclink voltage $V_{\mathrm{dc}}$. The $V_{\mathrm{g}} / V_{\mathrm{dc}}$ droop controller alters the ac-side power based on a measurement of $V_{\mathrm{dc}}$ by changing the amplitude of the inverters' terminal voltage $V_{\mathrm{g}}$, such that further dc-link voltage change is inhibited. Of course, the grid voltage should be kept inside the limits. Hence, the $P_{\mathrm{dc}} / V_{\mathrm{g}}$ droop controller changes the dc-side power $P_{\mathrm{dc}}$ based on the value of $V_{\mathrm{g}}$. A 


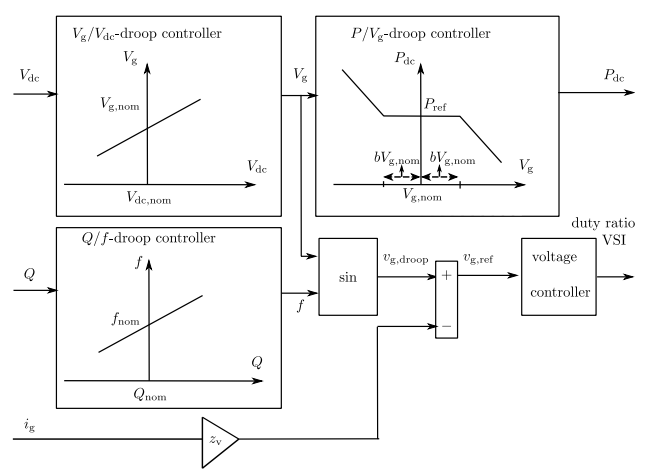

Figure 1: VBD controller and virtual impedance loop

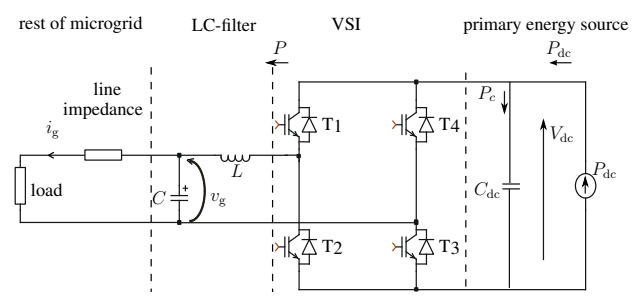

Figure 2: DG unit connected to the microgrid through a power-electronic interface, viz a voltage-source inverter (VSI). Parameters $\left(v_{\mathrm{g}}, i_{\mathrm{g}}, V_{\mathrm{dc}}, P, P_{\mathrm{dc}}\right)$

constant-power band on this change of $P_{\mathrm{dc}}$ is included as depicted in Fig. 1. This constant-power band is a voltage band in which the DG unit does not modify its output power based on the state of the microgrid. The parameter $2 b$ in in Fig. 1 determines the width of the constant-power band. In case the terminal voltage $V_{\mathrm{g}}$ of the DG unit is in the constant-power band, i.e., $(1-b) V_{\mathrm{g}, \text { ref }}<V_{\mathrm{g}}<(1+b) V_{\mathrm{g}, \text { ref }}$, the DG unit delivers its reference power $P_{\mathrm{dc}, \text { nom. }}$. Otherwise, the power of the DG unit is changed based on the state of the microgrid.

A distinction is made between dispatchable and less dispatchable DG units as illustrated in Fig. 3. For dispatchable DG units, analogously as for the central large generators, $P_{\text {ref }}$ represents the scheduled power that can be determined in the electricity markets. In this case, the value of $b$ is small, such that the unit reacts on small variations of the grid voltage.

For less dispatchable DG units, such as many renewables, a wider constantpower band is used. In this way, these units do not react on all load vari- 


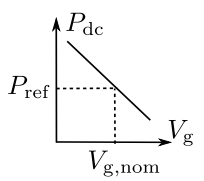

a) Fully controllable unit

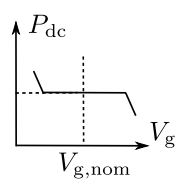

b) Less controllable unit

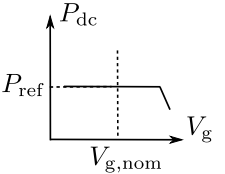

c) Renewable energy source

(without storage, nor controllable consumption)

Figure 3: Width of the constant-power band dependent on the characteristics of the considered DG unit

ations but change their output power only in case of more extreme voltage variations compared to the dispatchable DG units. These extreme voltages occur merely when the power limits of the dispatchable DG units are nearly reached. In these less dispatchable units, $P_{\text {ref }}$ can, for example, represent the instantaneous maximum power point of a wind turbine. Subsequently, an optimized integration of renewables is possible because the VBD control prioritizes the power changes of the units, by setting different values of $b$, without the need for communication.

Also, as the voltage is a local parameter, the voltage limits are sometimes already reached in areas where the dispatchable units are a minority compared to the less dispatchable ones. The current fit-and-forget strategy of integrating DG, solves these voltage problems by turning the DG units off. The latter may give rise to oscillation problems due to multiple switching of DG units and a significant loss of the renewable energy potential. In contrast, the VBD controller uses soft power curtailment to capture more of the renewable energy. In this way, voltage problems can be overcome as the renewables also take part in the voltage control [23].

For the reactive power sharing and the synchronization of the DG units, a $Q / f$ droop controller is used. As shown in Fig. 1 , the $V_{\mathrm{g}} / V_{\mathrm{dc}}$ and $Q / f$ droop controllers, which are digital controllers, determine the droop voltage $v_{\mathrm{g}, \mathrm{droop}, \mathrm{k}}=V_{\mathrm{g}, \mathrm{k}} \sin \left(\alpha_{\mathrm{k}}\right)$ ( $k$ is the discrete time instance and $\alpha$ the phase angle of the reference voltage). Discrete values are utilized because pulse width modulation with sampling period $T_{\mathrm{s}}$ is used in the converter. The amplitude $V_{\mathrm{g}}$ of the droop voltage is set by the $V_{\mathrm{g}} / V_{\mathrm{dc}}$ droop controller and the phase angle is obtained from the frequency $f$ in the $Q / f$ droop 


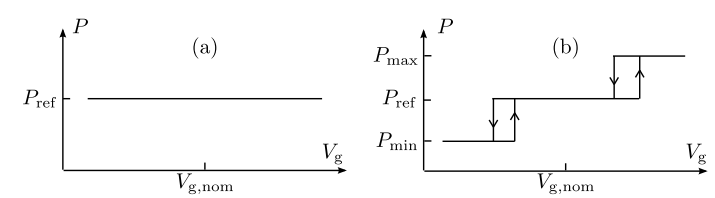

Figure 4: VBD control in the loads with constant-power band: active load with a primary load control based on the terminal voltage in a relay function

controller. Together, they determine

$$
v_{\mathrm{g}, \mathrm{droop}, \mathrm{k}}^{\star}=V_{\mathrm{g}, \mathrm{k}} \sin \left(\alpha_{\mathrm{k}-1}+2 \pi f_{\mathrm{k}} T_{s}\right)
$$

Fig. 1 also shows the virtual output impedance loop. A resistive output impedance $z_{v}=R_{v}$ is chosen as this provides more damping in the system $[18,19]$ and complies with the power control strategies of the loads and generators:

$$
v_{\mathrm{g}}^{\star}=v_{\mathrm{g}, \mathrm{droop}}^{\star}-R_{v} i_{g}
$$

with $v_{\mathrm{g}}^{\star}$ the reference voltage for the voltage controller, $v_{\mathrm{g}, \text { droop }}^{\star}$ the voltage obtained by the VBD controller and $i_{\mathrm{g}}$ the current injected into the microgrid, as illustrated in Fig. 2.

\subsection{VBD control of loads}

Because of the small scale of the microgrid and the large share of intermittent power sources, relying only on the DG units for the primary control may prove to be insufficient in islanded microgrids. Therefore, the loads are equipped with an analogous VBD control as the DG units [24]. Like in the DG units, the voltage is used to alter the consumption, with an example given in Fig. 4. Fig. 4(a) shows a conventional load, not acting on the state of the power system, while in Fig. 4(b), VBD control is included in this load, combined with a relay function for avoiding frequent switching. This is still a primary controller, operating fast and with negligible effects for the loads, e.g., a short-term (seconds) turning off a heating or ventilation system, or briefly changing the set temperatures of industrial freezers. A secondary controller will further deal with the specific load constraints, desires and the economic effect.

By also including constant-power bands in the load control, an automatic priority allocation can be set, e.g., (i) dispatchable DG units, (ii) controllable loads, (iii) less dispatchable DG units, (iv) other loads. The band is wider from category (i) to (iv). 


\section{Voltage-based droop control of storage}

Similar with the VBD control of the DG units and loads, in this paper, a VBD control is included in the storage elements as well. The VBD control has been developed for low-voltage microgrids which are characterized by mainly resistive microgrid lines inducing a linkage between active power $P$ and grid voltage amplitude $V_{\mathrm{g}}$. Hence, $P / V_{\mathrm{g}}$ droops instead of the frequency based control ( $P / f$ droop control) is used for the active power sharing between the units, including the storage elements, in the network. A generic storage element is considered and the control strategy is a primary control strategy. The main difference with the already-developed VBD control is that the state of charge (SOC) of the storage element needs to be taken into account.

An overview of the control layout is depicted in Fig. 5. The $V_{\mathrm{g}} / V_{\mathrm{dc}}$ droop and $Q / f$ droop controllers and the resistive virtual impedance $z_{\mathrm{v}}=R_{v}$, are the same as in the DG units. The $P_{\mathrm{dc}} / V_{\mathrm{g}}$ droop controller is however different in the storage elements. First, the storage elements are equipped with a $P_{\mathrm{dc}} / V_{\mathrm{g}}$ droop controller that determines the active power output of the storage element where $V_{\mathrm{g}, \text { ref }}$ instead of $V_{\mathrm{g}, \mathrm{nom}}$ determines the droop. Also, generally in storage elements $\overline{P_{\mathrm{dc}, \text { nom }}}$ equals $0 \mathrm{~W}$ as Fig. 5 shows:

$P_{\mathrm{dc}}=\left\{\begin{array}{l}P_{\mathrm{dc}, \text { nom }}-K_{\mathrm{P}}\left(V_{\mathrm{g}}-\left(V_{\mathrm{g}, \text { ref }}+b V_{\mathrm{g}, \text { nom }}\right) \quad \text { if } V_{\mathrm{g}}>V_{\mathrm{g}, \text { ref }}+b V_{\mathrm{g}, \text { nom }}\right. \\ P_{\mathrm{dc}, \text { nom }} \quad \text { if } V_{\mathrm{g}, \text { ref }}-b V_{\mathrm{g}, \text { nom }}<V_{\mathrm{g}}<V_{\mathrm{g}, \text { ref }}+b V_{\mathrm{g}, \text { nom }} \\ P_{\mathrm{dc}, \text { nom }}-K_{\mathrm{P}}\left(V_{\mathrm{g}}-\left(V_{\mathrm{g}, \text { ref }}-b V_{\mathrm{g}, \text { nom }}\right) \quad \text { if } V_{\mathrm{g}}<V_{\mathrm{g}, \text { ref }}-b V_{\mathrm{g}, \text { nom }}\right.\end{array}\right.$

The droop $K_{\mathrm{P}}$ is generally determined according to the ratings of the units, such that $\frac{P_{\mathrm{dc}, \text { nom }}}{K_{\mathrm{P}}}$ is equal for each DG unit. A constant-power band of width $2 b \cdot V_{\mathrm{g}, \text { nom }}$ is included, as depicted in Fig. 6(a). The first reason for implementing this band in the storage units is to allow for the normal variation of the grid voltage from its nominal value, i.e., between the voltage limits. The second reason is to allocate an automatic priority for the power changes between the different microgrid elements. The constant power band of the storage element is normally larger than that for controllable DG units, but smaller than for less controllable DG units and loads. In this way based on voltage changes, first, the controllable DG units react, e.g., by changing the fuel intake of a diesel genset. Next, storage elements react. If these are not sufficient, finally, the loads or less dispatchable DG units will alter their power exchange with the microgrid. Opposed to the loads and DG units, $P_{\text {ref }}$ of the storage element is equal to zero. The reference power can also be changed by a secondary, communication based controller that overlays the described primary VBD control strategy. This change can be based on 


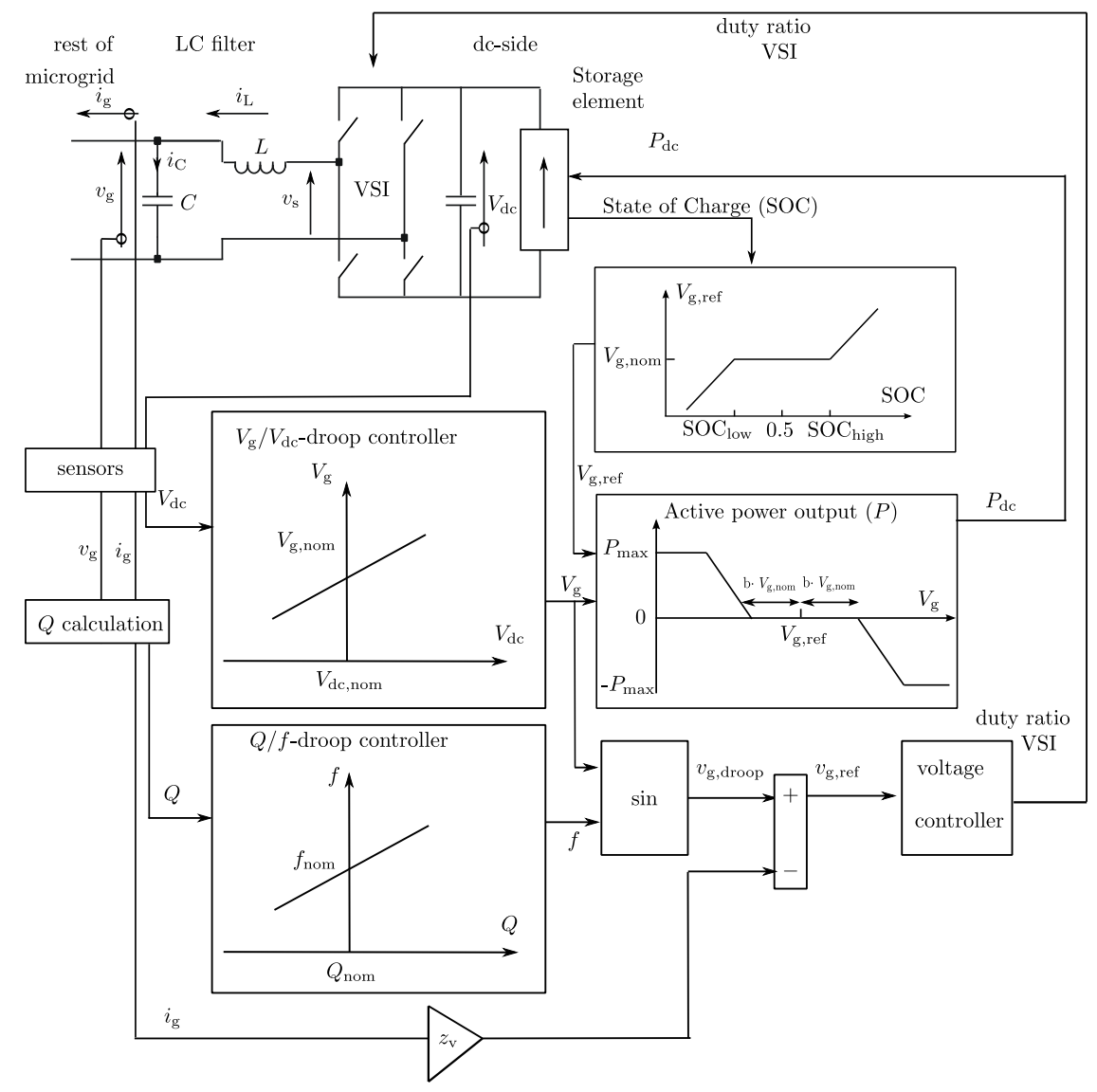

Figure 5: Control layout diagram

forecasts of the consumption/injection and market prices.

With $P_{\mathrm{dc}} / V_{\mathrm{g}}$ droop control with constant-power band, a static list in the reaction of the different units by setting the value of $b$ is imposed. The static listing can be dynamically varied by a slower, communication-based secondary controller changing $b$ of the units. However, for the storage element, the listing of this unit should be altered dynamically without inter-unit communication and based on its SOC, which is possible by the primary controller as it depends on local measurements only. For example a nearly empty storage element cannot react to voltage drops, which should be reflected in the control strategy. Therefore, second, the SOC of the unit is taken into account, with:

$$
S O C=\frac{\sum_{\mathrm{i}=1}^{N} P_{\text {exchange }} \Delta t}{E_{\max }}+\frac{E_{\text {initial }}}{E_{\max }} .
$$




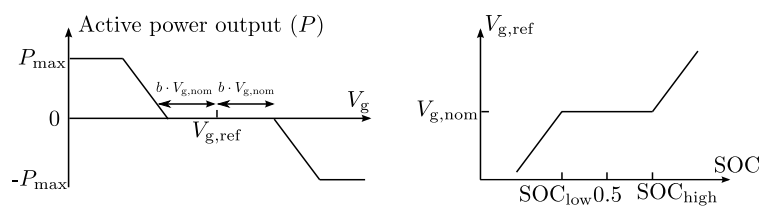

Figure 6: VBD control in the storage elements taking into account the SOC

This is done by making the $V_{\mathrm{g}, \text { ref }}$ parameter in the $P_{\mathrm{dc}} / V_{\mathrm{g}}$ droop controller dependent on the SOC. This is done by a $V_{\mathrm{g}, \mathrm{ref}} / \mathrm{SOC}$ curve as shown in Fig. 6(b):

$$
V_{\mathrm{g}, \text { ref }}=\left\{\begin{array}{l}
V_{\mathrm{g}, \text { nom }}+K_{\mathrm{S}}\left(S O C-S O C_{\mathrm{high}}\right) \quad \text { if } S O C>S O C_{\mathrm{high}} \\
V_{\mathrm{g}, \text { nom }} \quad \text { if } S O C_{\mathrm{low}}<S O C<S O C_{\mathrm{high}} \\
V_{\mathrm{g}, \text { nom }}-K_{\mathrm{S}}\left(S O C_{\mathrm{low}}-S O C\right) \text { if } S O C<S O C_{\mathrm{low}}
\end{array}\right.
$$

When the storage element is half full, i.e., SOC equals $0.5, V_{\mathrm{g}, \text { ref }}=V_{\mathrm{g}, \mathrm{nom}}$, with $V_{\mathrm{g}, \text { nom }}$ the nominal value of the considered microgrid, here $230 \mathrm{~V}$. In case the SOC of the storage element is low, the $V_{\mathrm{g}, \text { ref }} / \mathrm{SOC}$ curve lowers $V_{\mathrm{g}, \text { ref }}$. This shifts the $P_{\mathrm{dc}} / V_{\mathrm{g}}$ droop curve to the left, such that the delivery of power to the network in case of low terminal voltages is postponed to even lower voltages and the consumption of power is facilitated in case of a high grid voltage. High SOCs lead to a shift of the $P_{\mathrm{dc}} / V_{\mathrm{g}}$ droop curve to the right, facilitating the delivery of active power. Also, there is a dead band included in the $V_{\mathrm{g}, \text { nom }} / \mathrm{SOC}$ curve.

Third, some specific constraints of the storage element are taken into account. The SOC limits are taken into account by enforcing that if $S O C<$ $\mathrm{SOC}_{\text {low }}$ (e.g., 0.05$), P \leq 0$ ( $P$ smaller than zero indicates consumption of active power by the storage element) and vice versa, when $\mathrm{SOC}>\mathrm{SOC}_{\text {high }}$, $P \geq 0$, thus, injection of active power into the microgrid. An adequate value for $\mathrm{SOC}_{\text {low }}$ equals 0.3 and $\mathrm{SOC}_{\text {high }}$ equals 0.7 . The droop $K_{\mathrm{S}}$ is equal to 23 , following $K_{\mathrm{S}}=\frac{\Delta V_{\mathrm{g}}}{\Delta S O C}=\frac{10 \% V_{\mathrm{g}, \text { nom }}}{1}$. The droop $K_{\mathrm{P}}$ of the $P_{\mathrm{dc}} / V_{\mathrm{g}}$ droop controller is set in a similar manner, i.e., is generally determined according to the ratings of the units, such that $K_{\mathrm{P}}=\frac{P_{\mathrm{dc}, \text { nom }}}{\Delta V_{\mathrm{g}}}$. Here, the full power is injected for a voltage change of $10 \mathrm{~V}\left(=\Delta V_{\mathrm{g}}\right)$.

For the reactive power control, a $Q / f$ droop controller can be included, similar to that in the DG units. In this way, the storage element can also contribute in the reactive power sharing. 


\section{VBD storage control: transients study}

In the following simulation, the microgrid control in an islanded microgrid with one DG unit and one storage element is studied. First, the transient effects are studied in a simplified resistive microgrid. Next, inductance is included in the network lines showing a minimal impact.

The DG unit is modeled upto the level of the converter switches by using the PLECS library in MatLab/Simulink. The dc-side source of the DG units is simplified as a current source because a short-term transient study is made, i.e., the primary droop controller is studied. The difference in modeling for instance a PV panel and a diesel generator is the width of the constant-power band as generic DG units are used. The same is valid for the storage elements, i.e., generic elements are considered. Therefore, the dc-side is modeled as a current source, representing e.g., a battery (voltage source) behind a boost $\mathrm{dc} / \mathrm{dc}$ converter which is controlled as a current source. Details concerning energy storage modeling are given in [11]. The storage element starts loading $(P<0 \mathrm{~W})$ for high $V_{\mathrm{g}}$ and delivers power to the microgrid $(P>0 \mathrm{~W})$ is case of low $V_{\mathrm{g}}$. In this way, the storage element supports the microgrid. Helped by the usage of VBD control, high voltages occur for low load burden simultaneous with high renewable injection. As it is a primary controller, thus, without communication, the $P_{\mathrm{dc}} / V_{\mathrm{g}}$ droop controller enables the storage element to assist the microgrid by charging, hence, avoiding curtailment of the less dispatchale DG units. The charged power can again be injected into the microgrid in case of low grid voltages $V_{\mathrm{g}}$. Again, this is triggered without communication as low voltages indicate a high load burden simultaneous with low injection and the dispatchable DG units already injecting close to their limits.

The DG unit is connected through a line impedance of $0.5 \Omega$ to a load of $15 \Omega$, which in turn is connected to the storage element through a line impedance of $2 \Omega$. The transient effects after output changes of the DG unit are studied. From $0<t<0.4 \mathrm{~s}$, the nominal output power of the DG unit equals $2 \mathrm{~kW}$, from $0.4<t<0.8 \mathrm{~s}$ it increases to $2.5 \mathrm{~kW}$ and from $0.8<t<1.2 \mathrm{~s}$ it becomes $4.5 \mathrm{~kW}$. The DG unit is equipped with a large constant-power band with $b=8 \%$, e.g., representing a PV panel with a changing solar irradiation.

In the simulation results of Fig. 7, a storage element is included in the microgrid. This storage element has a maximum output power of $3 \mathrm{~kW}$ and has sufficient capacity such that the SOC is not affected significantly in this simulation. It operates without constant-power band. A small-scale microgrid is considered as large state variations can be imposed to clearly show the effects of the controllers. In the first time span, the terminal voltage of the storage element is below the nominal value, such that it contributes in the grid control by delivering active power to the microgrid. In the last time 


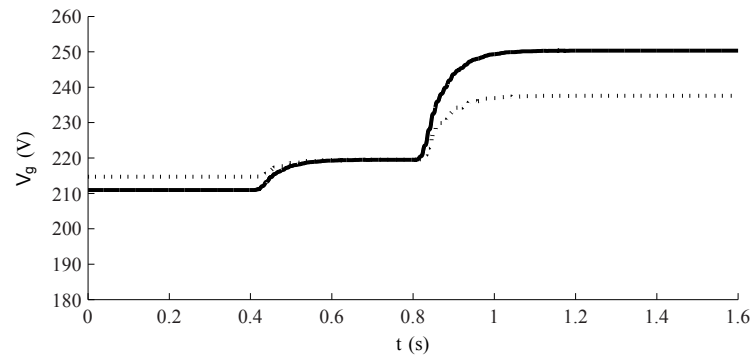

(a) Terminal voltage $V_{\mathrm{g}}$

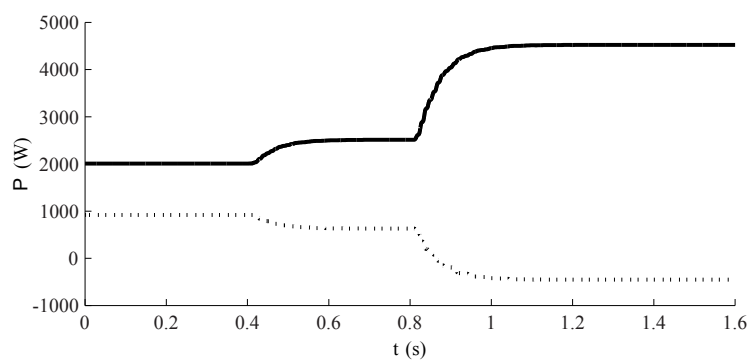

(b) Delivered power $P$

Figure 7: Small microgrid with transients study, actions of storage equipment $(-=$ DG unit, $\cdots=$ storage element)

period, the DG unit is delivering significantly more active power, such that the storage element starts charging.

In Fig. 7, relatively large voltage variations are observed. The reason is that a small microgrid without storage element is chosen. Also, the units are mainly inflexible. The primary controller studied here takes care of the transient effects, which are the focus of the paper. However, this primary controller is assisted by a secondary controller taking care of the energy management, thus, voltage management, in the islanded microgrid by dispatching and planning the microgrid assets.

Finally, in Fig 8, the line parameters $(R / X=3)$ and the load (load factor of $0.95)$ have an inductive part. The simulations show a minimal effect on the voltage, and virtually no transient effect. The same conclusion is drawn in $[12,24]$, hence, in the dynamics study of the following paragraph, an average model not taking into account inductive elements, can be used for studying longer simulation times. 


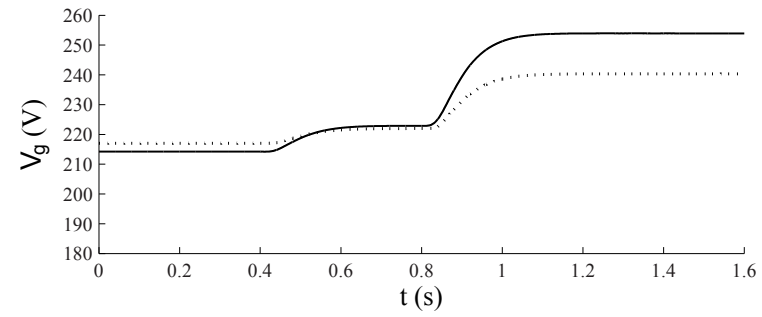

(a) Terminal voltage $V_{\mathrm{g}}$



(b) Delivered power $P$ and $Q$

Figure 8: Small microgrid with transients study, with storage equipment and inductive line parameters $\left(-=\mathrm{DG}\right.$ unit $\left(V_{\mathrm{g}}\right.$ and $\left.P\right), \cdots=$ storage element $\left(V_{\mathrm{g}}\right.$ and $\left.P\right)$, grayscale $-=\mathrm{DG}$ unit $(Q)$ and $-=$ storage element $(Q))$

\section{VBD storage control: dynamics study}

In the previous simulation, the SOC of the storage element did not change significantly because of the short simulation time. The reason is that for the transient study, the grid assets were modeled upto the converter switches in MatLab/Simulink, by using the PLECS library. In the following simulations, averaged models are used enabling longer simulation times. In averaged models, the storage elements and DG units are represented as voltage sources with voltage reference derived from the VBD control. Hence, opposed to the previous simulations, no switches and voltage controller are included. Only active power variations are studied (reactive power and frequency changes are studied above). The reason to leave out specific DG and storage details is that here, the interaction with the grid is considered in a generic manner. The time scales from storage/DG point of view are still short (16 s), from a grid's point of view they are large. First, a small microgrid is studied with significant changes in the SOC and next, a larger microgrid with more dynamic events.

\subsection{Slow dynamic changes}

The studied microgrid configuration is depicted in Fig. 9. Dynamic variations in the load and generation are included ('//' denotes 'in parallel with'), 


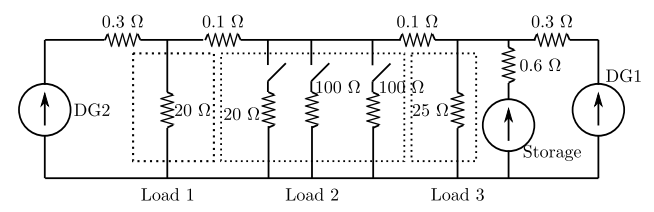

Figure 9: Configuration of studied microgrid: slow dynamic variations

these variations are summarized in Table 1. Three cases are compared. First,

Table 1: Dynamic variations in Fig. 9

\begin{tabular}{l|l|l} 
Unit & Time span & Value \\
\hline Load 1 & $0<t<16 \mathrm{~s}$ & $20 \Omega$ \\
\hline Load 2 & $0<t<3.5 \mathrm{~s}$ & $20 \Omega / / 20 \Omega / / 100 \Omega$ \\
& $3.5<t<5.0 \mathrm{~s}$ & $20 \Omega / / 100 \Omega / / 100 \Omega$ \\
& $5.0<t<16.0 \mathrm{~s}$ & $20 \Omega / / 100 \Omega$ \\
\hline Load 3 & $0<t<16 \mathrm{~s}$ & $25 \Omega$ \\
\hline \hline DG1 & $0<t<16 \mathrm{~s}$ & $P_{\text {ref }}=2 \mathrm{~kW}$ \\
\hline DG2 & $0<t<10 \mathrm{~s}$ & $P_{\text {ref }}=4 \mathrm{~kW}$ \\
& $10<t<16 \mathrm{~s}$ & $P_{\text {ref }}=6 \mathrm{~kW}$
\end{tabular}

a purely non flexible DG unit in a microgrid without storage is simulated. Next, the contribution of a storage element is analyzed. Finally, the impact of some flexibility of the DG unit is studied.

\subsubsection{No flexibility in storage element nor DG unit}

First, all DG units are undispatchable $(b=\infty)$ and no storage is included in Fig. 10. The voltage limits are exceeded for a large amount of time, i.e., $10<t<16 \mathrm{~s}$. This illustrates the need for flexibility.

\subsubsection{Flexibility in storage element not in $D G$ unit}

Therefore, in Fig. 11, a storage element with $b=0 \%$ is included, with a maximum output/input power of $4 \mathrm{~kW}$ and a maximum capacity of $10 \mathrm{mAh}$ (i.e., a very small storage element in order to magnify and fasten the effect of the SOC changes). The initial SOC equals 0.5. The voltage limits $\left( \pm 10 \% V_{\mathrm{g}, \text { nom }}\right)$ are exceeded for a smaller amount of time, i.e., $12.1<t<16 \mathrm{~s}$. This illustrates the need to properly tune the flexibility of all grid elements (constant power bands of DG units, loads and storage elements as well as the storage buffer). In the first time span $0<t<1.4 \mathrm{~s}$, 


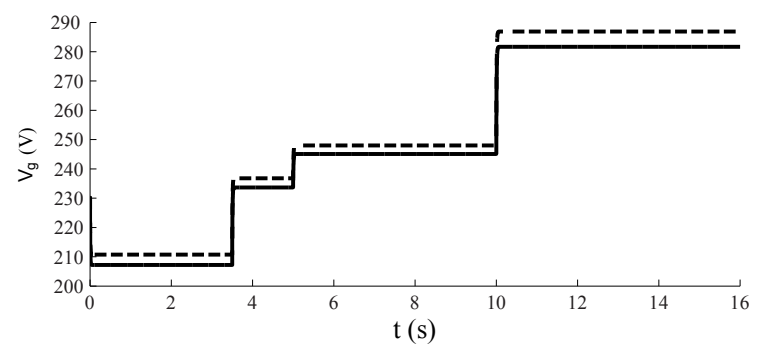

(a) Terminal voltage $V_{\mathrm{g}}$

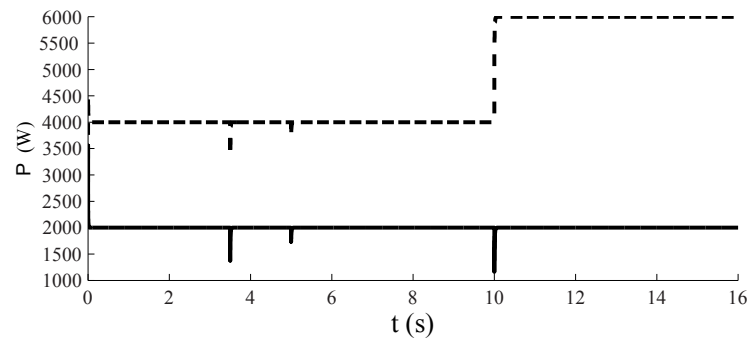

(b) Delivered power $P$

Figure 10: Microgrid with dynamic changes, without actions of storage equipment, undispatchable DG units (- = DG1, - - = DG2)

the SOC of the storage element is between 0.3 and 0.7 , which is the dead band of the $V_{\mathrm{g}, \text { nom }} / \mathrm{SOC}$ curve $\left(\mathrm{SOC}_{\text {low }}=0.3\right.$ and $\mathrm{SOC}_{\text {high }}=0.7$ in Fig. 6 ). Hence, $V_{\mathrm{g}, \text { ref }}=V_{\mathrm{g} \text {,nom }}$ and remains constant. As in this time span, the terminal voltage of the storage element is slightly under its nominal value, the storage element delivers power to the network. Next, the SOC drops below 0.3 , leading to a decreasing $V_{\mathrm{g}, \text { ref }}\left(V_{\mathrm{g}, \mathrm{ref}}<V_{\mathrm{g}, \mathrm{nom}}\right)$, proportionally with SOC. This shifts the $P_{\mathrm{dc}} / V_{\mathrm{g}}$ droop curve to the left, decreasing the power delivery. At $t=3.5 \mathrm{~s}$, the load burden decreases, which increases the terminal voltages. As $V_{\mathrm{g}, \text { storage }}<V_{\mathrm{g}, \text { ref }}$, the storage element starts charging. At $t=5 \mathrm{~s}$ the load decreases even more, leading to an increased consumption of the storage equipment. At $t=6.7 \mathrm{~s}$, the rate of charging the storage element decreases as the SOC again reaches the 0.3 value, hence, $V_{\mathrm{g}, \text { ref }}=V_{\mathrm{g}, \text { nom }}$. From $t=11.5 \mathrm{~s}$ on, the storage element is full, losing the flexibility for the voltage control in the microgrid.

\subsubsection{Flexibility in both storage element and $D G$ unit}

When some flexibility is included in the DG units as well, the voltage limits are not reached. For instance, DG1 is a slightly controllable DG unit with constant-power band of $b=8 \%$. DG2 is more dispatchable with $b=$ 


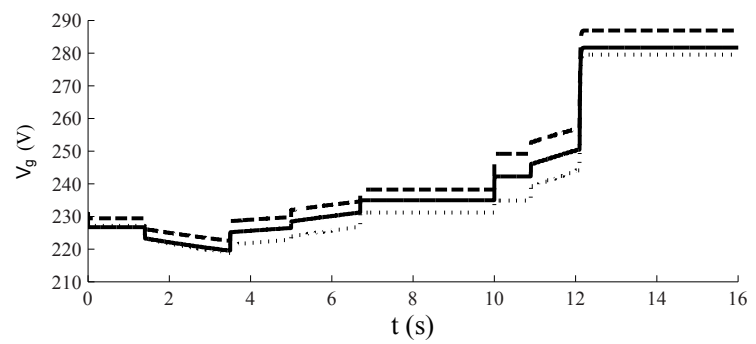

(a) Terminal voltage $V_{\mathrm{g}}$

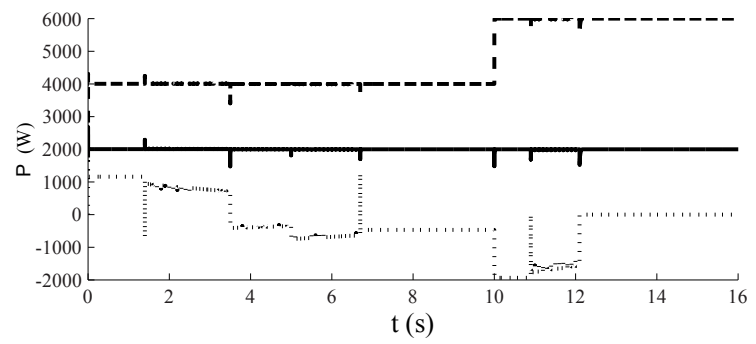

(b) Delivered power $P$

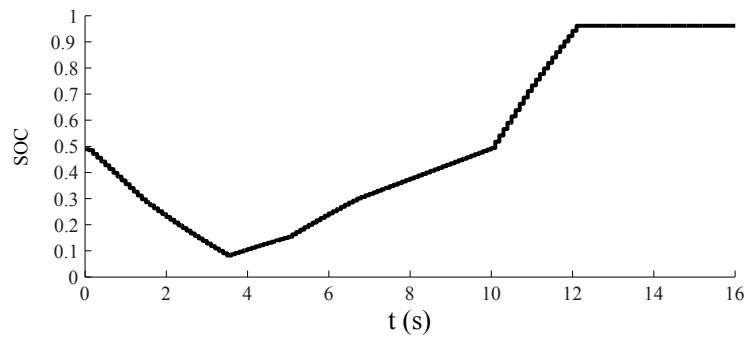

(c) State of charge (SOC)

Figure 11: Microgrid with dynamic changes, actions of storage equipment, undispatchable DG units ( $-=$ DG1, - - - = DG2, $\cdots=$ storage element)

$5 \%$. Hence, although this remains a microgrid that is to a large extend still inflexible, the combination of storage and some flexibility in the generations significantly improves the voltage quality. Both units have a droop $K_{\mathrm{P}}=$ $P_{\text {nom }} / 10$. In this case, only DG2 needs to react, i.e., $P<P_{\text {ref }}=6 \mathrm{~kW}$ in the last time spans to avoid voltage limit violation.

\subsubsection{Summary}

The simulations show that the storage element alone has already a significant positive effect on the microgrid operation. The cooperation between the storage element and flexible DG units, through VBD control, helps avoiding voltage limit violation with a minimal curtailment of the renewable energy 
sources. Also, the VBD controllers of the DG unit and the storage element operate appropriately together in assuring a reliable microgrid operation, priority in activation of output power changes and in voltage control. Even better results would be obtained when including a slow communicationbased controller. The storage element's VBD controller reacts as desired, i.e., the reaction of the storage element is based on its SOC as imposed by the droop in Fig. 6b; and based on the terminal voltage as imposed by Fig. 6(a).

\subsection{Fast dynamic changes}

In the following simulation, some faster dynamical events are included in order to study the effect of changing parameters in an islanded microgrid including a storage element, flexible DG units and flexible loads (demand dispatch). Two cases are compared, i.e., with and without storage element. The microgrid configuration of Fig. 12 is studied. The main microgrid parameters are detailed in the table of Fig. 12. This is the same microgrid as in [24], here with inclusion of storage. This microgrid consists of three power sources DG1, DG2 and DG3, the details of which are given in Fig. 12. The nominal generated dc currents are: $I_{\mathrm{dc}, 1}=12 \mathrm{~A}, I_{\mathrm{dc}, 2}=8 \mathrm{~A}$ and $I_{\mathrm{dc}, 3}=5 \mathrm{~A}$. The loads considered here are a combination of variable and non-variable loads. The simulations mimic a dynamic profile, with dynamic events such as losing a generator (DG2) and changing the demand dispatch $\left(R_{\mathrm{var}, 1}\right.$ and $\left.R_{\mathrm{var}, 2}\right)$. Further details concerning the demand dispatch strategy are presented in [24].

Note that the transients follow very quickly. The reason is to have short simulation times, as the steady-state conditions give no further information. Starting from $230 \mathrm{~V}$ without demand dispatch:

- $t=0$ s: all DG units operate at $100 \%$ (nominal) current;

- $t=0.35$ s: DG3 switches off;

- $t=0.36 \mathrm{~s}$ : start of the demand dispatch for both variable loads according to the previous case. The demand dispatch operates with a delay, for stability reasons, to include measurement and responsive delay and for practical reasons in the implementation in the loads. $R_{\mathrm{var}, 1}$ can change from $25 \Omega$ to $50 \Omega$ when the terminal voltage $V_{\mathrm{g}}$ drops below $0.91 V_{\mathrm{g}, \text { nom }}$ and back to $25 \Omega$ when it again exceeds $0.96 V_{\mathrm{g}, \text { nom }}$ in the relay function of Fig. 4. The second load with demand dispatch $R_{\mathrm{var}, 2}$ can change from $25 \Omega$ to $5000 \Omega$ (i.e., turned off) when $V_{\mathrm{g}, \mathrm{o}} \leq 0.93 \cdot V_{\mathrm{g}, \text { nom }}$ and back to $25 \Omega$ when $V_{\mathrm{g}, \mathrm{d}}>0.97 \cdot V_{\mathrm{g}, \mathrm{nom}}$.

- $t=0.70 \mathrm{~s}$ : DG3 starts operating at $50 \%$;

- $t=0.85 \mathrm{~s}$ : demand dispatch turns off for the first variable load, such that $R_{\mathrm{var}, 1}=25 \Omega$, as, e.g., its power consumption can not be delayed any further, then, $R_{\mathrm{var}, 1}=25 \Omega$.

- $t=1 \mathrm{~s}$ : the nominal current of DG2 decreases to $75 \%$, i.e., $I_{\mathrm{dc}, 2, \text { nom }}=6$ A. 


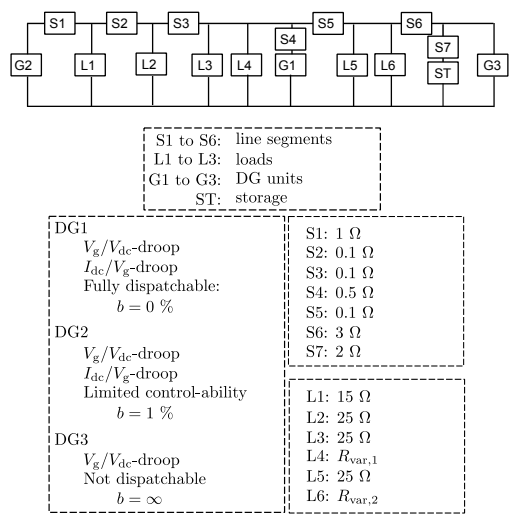

Figure 12: Microgrid configuration: extended example

The storage unit has a maximum output power of $4 \mathrm{~kW}$, which it can deliver during $0.4 \mathrm{~s}$ (i.e., a small capacity of the storage element for decreasing the simulation times). Its initial SOC equals 0.5 and the SOC measurements are updated each $0.1 \mathrm{~s}$.

The simulation results are depicted in Fig. 13. The SOC remains between 0.48 and 0.58 .

- $0<t<0.35$ s: After a start-up transient, the terminal voltages reach $V_{\mathrm{g}, 1}=221 \mathrm{~V}, V_{\mathrm{g}, 2}=224 \mathrm{~V}, V_{\mathrm{g}, 3}=240 \mathrm{~V}$ and for the storage element $V_{\mathrm{g}, \text { stor }}=232 \mathrm{~V}$. Hence, the storage element charges.

- $0.35<t<0.7 \mathrm{~s}$ : The demand signal for both variable loads turns on. Despite the loss of DG3, the contribution of the demand dispatch leads to a small voltage increase to $V_{\mathrm{g}, 1}=226 \mathrm{~V}, V_{\mathrm{g}, 2}=228 \mathrm{~V}, V_{\mathrm{g}, 3}=$ $240 \mathrm{~V}$. As the storage element is connected near the DG unit (DG3) which is turned off, the voltage at this location slightly decreases, i.e., $V_{\mathrm{g}, \text { stor }}=228 \mathrm{~V}$. Therefore, the storage element changes its state from consuming to delivering some active power to the microgrid.

- $0.7<t<0.85$ s: Higher microgrid voltages are obtained because of the activation of DG3. Hence, the storage element's delivered power decreases slightly.

- $0.85<t<1 \mathrm{~s}$ : At $0.85 \mathrm{~s}$, the demand signal of $R_{\mathrm{var}, 1}$ is turned off. The load turns back on, leading to a higher load burden. Therefore, the microgrid voltages are slightly decreased and DG1 and DG2 respond by increasing their output current. The storage element decreases its power consumption. 


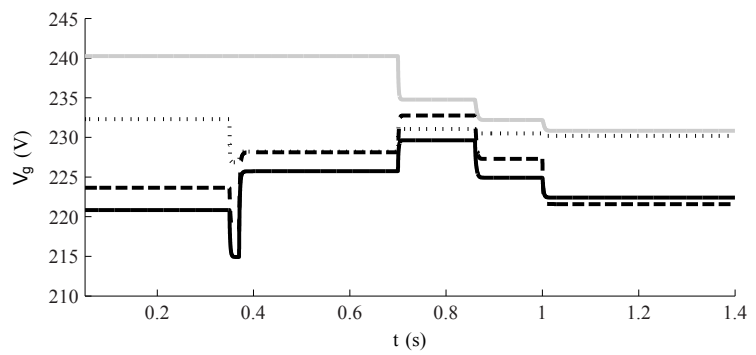

(a) Terminal voltage $V_{\mathrm{g}}$

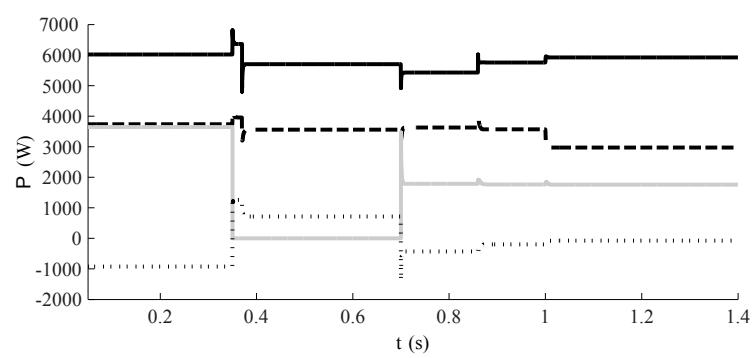

(b) Delivered power $P$

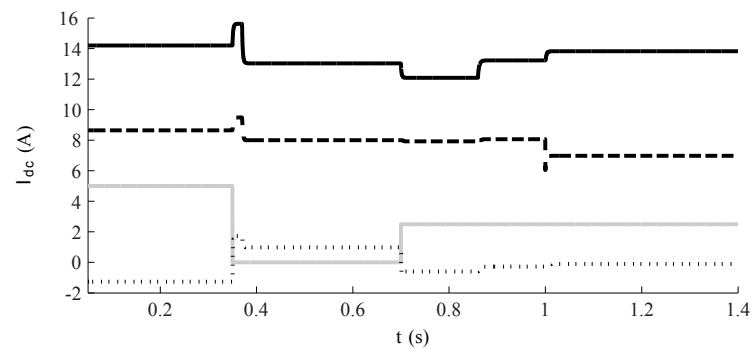

(c) Delivered dc-current $I_{\mathrm{dc}}$

Figure 13: Microgrid with fast dynamic changes, actions of storage equipment and (slightly) controllable DG units $(-=\mathrm{DG} 1,-\cdots=\mathrm{DG} 2,-($ grayscale $)=\mathrm{DG} 3, \cdots$ $=$ storage element)

- $t>1 \mathrm{~s}$ : Because less power is delivered by DG2, the microgrid voltages are lower compared to the previous time span: with $V_{\mathrm{g}, 1}=222.4 \mathrm{~V}$, $V_{\mathrm{g}, 2}=221.6 \mathrm{~V}, V_{\mathrm{g}, 3}=230.8 \mathrm{~V}$ and $V_{\mathrm{g}, \mathrm{stor}}=230.2 \mathrm{~V}$. Furthermore, because of the lower output current of DG2, the output current of DG1 increases $\left(I_{\mathrm{dc}} / V_{\mathrm{g}}\right.$ droop control). In steady-state also, $I_{\mathrm{dc}, 1}=13.8 \mathrm{~A}$, $I_{\mathrm{dc}, 2}=7.0 \mathrm{~A}, I_{\mathrm{dc}, 3}=2.5 \mathrm{~A}$ and $I_{\mathrm{dc}, \mathrm{stor}}=-0.1 \mathrm{~A}$.

This simulation illustrates the contribution of the storage element in the microgrid control. For comparison, the case without contribution of the storage element is depicted in Fig. 14. In this case, more extreme voltages and pos- 


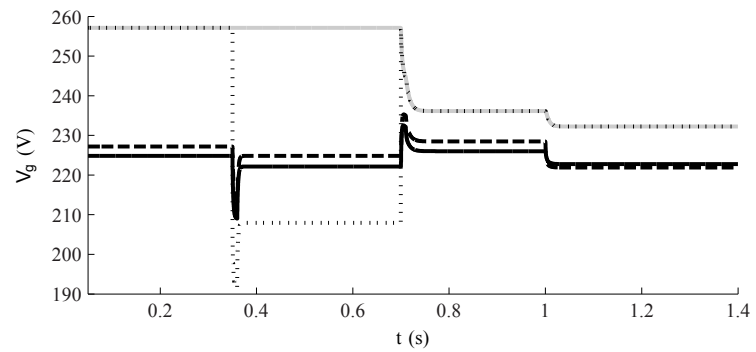

(a) Terminal voltage $V_{\mathrm{g}}$

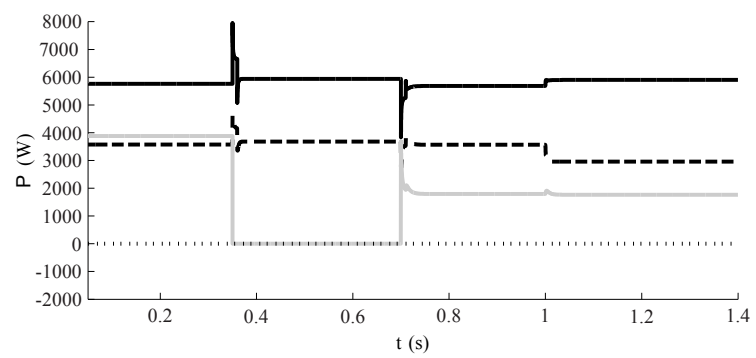

(b) Delivered power $P$

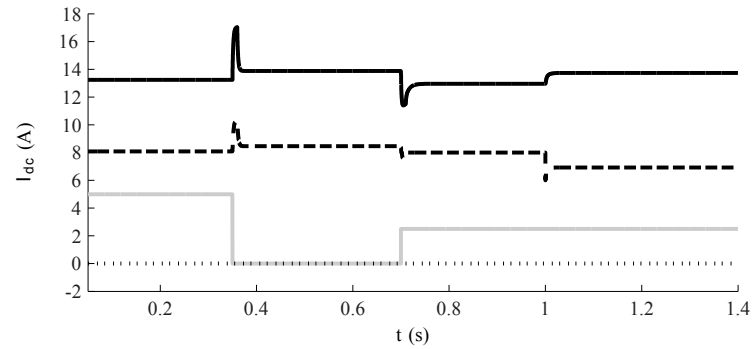

(c) Delivered dc-current $I_{\mathrm{dc}}$

Figure 14: Microgrid with fast dynamic changes, without actions of storage equipment and (slightly) controllable DG units $(-=$ DG1, - - = DG2, - (grayscale $)=$ DG3, $\cdots$ $=$ storage element)

sible over and under voltages can be reached. For instance, in the first time span, by consuming, the storage element is effective in decreasing the voltage near DG3. This shows that certainly in small-scale microgrids with limited demand response and a significant share of renewables, storage can largely improve the voltage quality. 


\section{Experimental results}

The experimental microgrid set-up consists of two voltage-source inverter (VSI) interfaced DG units and various loads. The inverters have been realized by using a printed circuit board that was developed at Ghent University. In the experiments described here, this inverter is applied in a single-phase configuration by using a full-bridge. The dc-side of the inverter, i.e., the energy source, is emulated as a dc current source by means of the Sorensen SGI6000/17C source. In the experiments, the islanded microgrid consists of two DG units connected to the same load through line impedances. The measurements are digitized by using 12-bit analogue-to-digital converters (ADCs). An FPGA Spartan 3E 1600 is used for determining the PWM signals.

\subsection{VBD control of the $D G$ units}

Table 2: Active power sharing of 2 DG units

\begin{tabular}{l|l|c|l||l|l|l|l} 
case & $\begin{array}{c}\text { load } \\
(\Omega)\end{array}$ & unit $(\mathrm{DG})$ & $\begin{array}{l}b \\
(\%)\end{array}$ & $\begin{array}{l}I_{\mathrm{dc}} \\
(\mathrm{A})\end{array}$ & $\begin{array}{l}V_{\mathrm{dc}} \\
(\mathrm{V})\end{array}$ & $\begin{array}{l}V_{\mathrm{g}} \\
(\mathrm{V})\end{array}$ & $\begin{array}{l}P \\
(\mathrm{~W})\end{array}$ \\
\hline \hline 1 & 27 & 1 & $\infty$ & 1 & 186 & 146 & 183 \\
& & 2 & $\infty$ & 1 & 188 & 148 & 183 \\
2 & 13 & 1 & $\infty$ & 1 & 120 & 80 & 111 \\
& & 2 & $\infty$ & 1 & 121 & 81 & 107 \\
3 & \multirow{2}{*}{13} & 1 & $\infty$ & 2 & 181 & 141 & 340 \\
& & 2 & $\infty$ & 2 & 184 & 144 & 342 \\
\hline 4 & 27 & 1 & 0 & 1.5 & 213 & 173 & 300 \\
& & 2 & $\infty$ & 1 & 212 & 172 & 206 \\
5 & 13 & 1 & 0 & 2.8 & 181 & 141 & 471 \\
& & 2 & $\infty$ & 1 & 170 & 120 & 160
\end{tabular}

Table 2 illustrates the power sharing between the two DG units for varying loads, constant power bands and dc-current of the DG units. The nominal ratings of the units are $I_{\mathrm{dc}, \text { nom }}=2 \mathrm{~A}, V_{\mathrm{dc}, \mathrm{nom}}=200 \mathrm{~V}$ and $V_{\mathrm{g}, \mathrm{nom}}=160 \mathrm{~V}$ amplitude. The DG units are operated as current sources at the dc-side and the effect of changing load and dc current is studied. When comparing cases $1-2$, the units deliver the same $I_{\mathrm{dc}}$ for a variable load showing that $V_{\mathrm{g}}$ is altered when the load changes, i.e., according to the $V_{\mathrm{g}} / V_{\mathrm{dc}}$ droop controller. Here, if the load increases (the dc-link voltage decreases), the output power decreases. This is because the DG units are modeled as constant dc-current sources. For instance, for wind turbines and photovoltaic panels, this is a valid assumption for transients, i.e., the primary control considered here. 
For larger time frames, they are constant-power sources (as long as solar irradiation or wind power remains equal), hence, $I_{\mathrm{dc}}$ alters when $V_{\mathrm{dc}}$ changes. In cases 1-3, units with $b=\infty$ are used, hence, with a fixed $I_{\mathrm{dc}}$. Here, the delivered dc-current $I_{\mathrm{dc}}$ equals a pre-set value. In case 2 , the load has significantly increased. This is clearly visible in the lower grid voltage because of the large constant-power band. In the case 3, the larger output powers of the DG units lead to higher microgrid voltages compared to case 1.

In the cases $4-5$, DG1 is dispatchable $(b=0 \%)$, while DG2 remains with large (here, infinite) constant-power band. Hence, the output current of DG1 is determined by the $I_{\mathrm{dc}} / V_{\mathrm{g}}$ droop controller, which is equivalent with the $P_{\mathrm{dc}} / V_{\mathrm{g}}$ droop controller, thus, $I_{\mathrm{dc}, 1}=I_{\mathrm{dc}, 1, \mathrm{droop}}$, and $I_{\mathrm{dc}, 2}=I_{\mathrm{dc}, 2, \text { nom }}$. When comparing the cases, indeed DG1 captures the changing load by altering the output $I_{\mathrm{dc}}$. Compared to case 2 , in case 5 , the voltage is clearly closer to its nominal value of $160 \mathrm{~V}$ because of the presence of a dispatchable DG unit. In conclusion, the active power is shared according to the ratings of the DG units. For undispatchable DG units, the terminal voltage is altered to deliver the available power to the islanded microgrid. Dispatchable DG units alter their input power $\left(I_{\mathrm{dc}}\right)$ to force the voltage closer to its nominal value.

\subsection{Storage control}

Next, one undispatchable DG unit and a storage element are connected to the microgrid set-up. The storage element has been implemented as a dcbattery emulator connected to the dc-link of an inverter of the same type as that of the DG unit. Here, large droops (thus, not taking into account voltage limits) are included to clearly show the effect of changing parameters. The DG unit and the storage element are connected to the load of $27 \Omega$ through a line resistance of $3.6 \Omega$ each. The measurement results are summarized in Table 3.

For the cases 1 to 4, the DG units' output power has increased. If the

Table 3: DG unit (DG) and storage element (ST)

\begin{tabular}{ll||l|l|l|l} 
case & & 1 & 2 & 3 & 4 \\
\hline \multirow{2}{*}{$V_{\mathrm{g}}(\mathrm{V})$} & $\mathrm{DG}$ & 108 & 147 & 188 & 203 \\
& $\mathrm{ST}$ & 83 & 113 & 144 & 157 \\
\hline \multirow{2}{*}{$P(\mathrm{~W})$} & $\mathrm{DG}$ & 284 & 526 & 868 & 1020 \\
& $\mathrm{ST}$ & -78 & -153 & -249 & -299
\end{tabular}

terminal voltage would not alter, this results in an increasing $V_{\mathrm{dc}}$. Because of the $V_{\mathrm{g}} / V_{\mathrm{dc}}$ droop controller and the large constant power band, $V_{\mathrm{g}}$ increases to deliver the available power to the network. The storage element senses this increasing terminal voltage and increases its charging from cases 1 to 4 . 
In Table 4, the same configuration is measured, but the storage element has a lower rating (lower $P_{\max }$ in Fig. 6). The results show that for the same voltage (e.g., comparing cases 2 in Table 3 and Table 4), the consumption (negative $P$ ) of the storage element is lower such that the DG unit can deliver less power (or even needs to be curtailed more). Also, for the same active power output of the DG unit, the storage element consumes less in Table 4, resulting in higher terminal voltages.

Table 4: DG unit (DG) and smaller storage element (ST)

\begin{tabular}{ll||l|l|l|l|l} 
& & 1 & 2 & 3 & 4 & 5 \\
\hline \multirow{2}{*}{$V_{\mathrm{g}}(\mathrm{V})$} & DG & 103 & 125 & 145 & 187 & 246 \\
& ST & 95 & 111 & 128 & 157 & 180 \\
\hline \multirow{2}{*}{$P(\mathrm{~W})$} & DG & 137 & 239 & 354 & 655 & 917 \\
& ST & 35 & 13 & -16 & -80 & -159
\end{tabular}

\section{Conclusion}

This paper presents a voltage-based droop control for the storage elements in islanded microgrids. This storage controller adequately cooperates with a similar voltage-based droop control of the other microgrid elements, such as the controllable loads and the DG units. As the microgrid control is without communication and the storage elements assist in the microgrid control in a coherent manner with the other grid assets, this strategy benefits the microgrid stability and makes for a consistent control strategy. Because of this consistency, it enables a swift implementation and comprehension in managing the microgrid in practice. Some simulations are discussed, firstly showing the transient effect of the control strategy. Secondly, more extensive simulations show the voltage-triggered reaction of the controllers of the loads, generators and the storage elements on varying dynamical simulations. Finally, an experimental validation is presented concerning the VBD control for DG units and its cooperation with storage control. This paper illustrates that using the terminal voltage for the primary control is effective to enable a proper cooperation of the different grid assets in the microgrid control with a similar control strategy and without inter-unit communication. 


\section{Acknowledgments}

The research was carried out in the frame of the Inter-university Attraction Poles program IAP-VII-02, funded by the Belgian Government. The research of T. Vandoorn and J. D. M. De Kooning is funded by the Special Research Fund (BOF) of Ghent University (Belgium) with a post-doctoral and a $\mathrm{PhD}$ fellowship respectively.

\section{References}

[1] T. Malakar, S. K. Goswami, A. K. Sinha, Optimum scheduling of a micro grid connected wind-pumped storage hydro plant in a frequency based pricing environment, Electrical Power and Energy Systems 54 (2014) 341-351.

[2] S. Youli, Z. Litifu, K. Nagasaka, Efficiency of micro grid with storage battery in reliability, economy and environment assessments, Electrical Power and Energy Systems 3 (2009) 154-162.

[3] Energy storage systems providing primary reserve and peak shaving in small isolated power systems: An economic assessment, International Journal of Electrical Power \& Energy Systems 53 (2013) 675 - 683.

[4] J. K. Kaldellis, D. Zafirakis, E. Kondili, Optimum sizing of photovoltaic-energy storage systems for autonomous small islands, Internat. Journal of Electrical Power and Energy Systems 32 (2010) 2436 .

[5] A new approach for optimal sizing of battery energy storage system for primary frequency control of islanded microgrid, Internat. Journal of Electrical Power and Energy Systems 54 (2014) 325 - 333.

[6] H. Farhangi, The path of the smart grid, IEEE Power \& Energy Magazine 8 (2010) 18-28.

[7] R. H. Lasseter, P. Paigi, Microgrid: A conceptual solution, in: Proc. IEEE Power Electron. Spec. Conf. (PESC 2004), volume 6, Aachen, Germany, pp. 4285-4298.

[8] J. Rocabert, A. Luna, F. Blaabjerg, P. Rodrígez, Control of power converters in AC microgrids, IEEE Trans. Power Electron. 27 (2012) $4734-4748$.

[9] F. Katiraei, M. R. Iravani, P. W. Lehn, Micro-grid autonomous operation during and subsequent to islanding process, IEEE Trans. Power Del. 20 (2005) 248-257. 
[10] M. Gauthier, C. Abbey, F. Katiraei, J.-L. Pepin, M. Plamondon, G. Simard, Planned islanding as a distribution system operator tool for reliability enhancement, in: $19^{\text {th }}$ International Conference on Electricity Distribution, Vienna.

[11] X. Tan, Q. Li, H. Wang, Advances and trends of energy storage technology in microgrid, Internat. Journal on Electrical Power and Energy Systems 44 (2013) $179-191$.

[12] T. L. Vandoorn, B. Meersman, L. Degroote, B. Renders, L. Vandevelde, A control strategy for islanded microgrids with dc-link voltage control, IEEE Trans. Power Del. 26 (2011) 703-713.

[13] J. M. Guerrero, M. Chandorkar, T.-L. Lee, P. C. Loh, Advanced control architectures for intelligent microgrids - Part I: Decentralized and hierarchical control, IEEE Trans. Ind. Electron. 60 (2013) 1254-1262.

[14] T. L. Vandoorn, J. D. M. De Kooning, B. Meersman, L. Vandevelde, Review of primary control strategies for islanded microgrids with powerelectronic interfaces, Renewable and Sustainable Energy Reviews 19 (2013) 613-628.

[15] M. C. Chandorkar, D. M. Divan, R. Adapa, Control of parallel connected inverters in standalone ac supply systems, IEEE Trans. Ind. Appl. 29 (1993) 136-143.

[16] M. Marwali, A. Keyhani, Control of distributed generation systems part i: Voltages and current control, IEEE Trans. Power Electron. 19 (2004) 1541-1550.

[17] Y. Mohamed, E. F. El-Saadany, Adaptive decentralized droop controller to preserve power sharing stability for paralleled inverters in distributed generation microgrids, IEEE Trans. Power Electron. 23 (2008) $2806-2816$.

[18] J. M. Guerrero, J. Matas, L. García de Vicuña, M. Castilla, J. Miret, Decentralized control for parallel operation of distributed generation inverters using resistive output impedance, IEEE Trans. Ind. Electron. 54 (2007) 994-1004.

[19] W. Yao, M. Chen, J. M. Guerrero, Z.-M. Qian, Design and analysis of the droop control method for parallel inverters considering the impact of the complex impedance on the power sharing, IEEE Trans. Ind. Electron. 58 (2011) 576-588. 
[20] Y. W. Li, C. N. Kao, An accurate power control strategy for power electronics interfaced distributed generation units operating in a low voltage multibus microgrid, IEEE Trans. Power Electron. 24 (2009) 2977-2988.

[21] A. Engler, O. Osika, M. Barnes, N. Hatziargyriou, DB2 Evaluation of the local controller strategies, www.microgrids.eu/micro2000, 2005.

[22] C. Sao, P. Lehn, Control and power management of converter fed microgrids, IEEE Trans. Power Syst. 23 (2008) 1088-1098.

[23] T. L. Vandoorn, J. D. M. De Kooning, B. Meersman, L. Vandevelde, Voltage-based droop control of renewables to avoid on-off oscillations caused by overvoltages, IEEE Trans. Power Del. 28 (2013) 845-854.

[24] T. L. Vandoorn, B. Renders, L. Degroote, B. Meersman, L. Vandevelde, Active load control in islanded microgrids based on the grid voltage, IEEE Trans. on Smart Grid 2 (2011) 139-151. 